\title{
Ranked ordering sources and embedded modality
}

\author{
Drew Reisinger*
}

\begin{abstract}
I present an ordering semantics for modality in which possible worlds are ordered by ordering sources augmented with a partial order structure. This extension of Kratzer's (1991) ordering semantics allows propositions to contribute to the ideal defined by an ordering source with differing degrees of priority and allows this priority relation to vary with the world of evaluation. Although the * operator of Katz et al. (2012) also allows ordering sources to be combined with different degrees of priority, I show that it does not account for a variant of Goble's (1996) Medicine Problem in which a modal is embedded under an attitude verb. I also extend the investigation by Katz et al. (2012) into the combinatorial structure of complex ordering sources by proposing a generalization of their * operator for partially ordered ordering sources.
\end{abstract}

Keywords. modality; semantics; ordering semantics; intensional semantics; formal semantics; discourse context

1. Introduction. In this paper, I present the Embedded Medicine Problem, an extension of Goble's (1996) Medicine Problem in deontic logic that demonstrates the need for ordering source priorities that can be shifted by intensional operators. I show how ordering sources augmented with a partial order structure, which I will call ranked ordering sources, meet this need, and I present a mechanism by which ranked ordering sources induce orderings on possible worlds.

This paper is structured as follows. In section 2, I review some of the relevant work on modality and ordering semantics, focusing particularly on the mechanism of Katz et al. (2012) for combining ordering sources with differing degrees of priority and how it solves Goble's (1996) Medicine Problem. In section 3, I introduce the Embedded Medicine Problem and show that it presents difficulties for the Katz et al. (2012) account. In section 4, I describe the formal machinery of ranked ordering sources and show how my proposal addresses the difficulties raised by the Embedded Medicine Problem. In section 5, I relate ranked ordering sources to the research program introduced by Katz et al. (2012) of investigating mechanisms for constructing complex ordering sources from simpler ones, and I define a combinatorial operator on ordering sources, the priority join, that generalizes the * operator from Katz et al. (2012). Finally, in section 6, I conclude by speculating on the application of ranked ordering sources to various linguistic phenomena addressed by the modality literature.

2. Background. In this section, I first briefly review Kratzer's (1991) now standard doubly relative semantics for modality. I then present Goble's (1996) Medicine Problem, which challenges the standard approach. Finally, I describe the mechanism proposed byKatz et al. (2012) for combining ordering sources with different degrees of priority and show how it solves the Medicine Problem.

\footnotetext{
* I would like to thank Kyle Rawlins for his extensive mentorship in every phase of this project and Paul Smolensky for his invaluable insights on applying Optimality Theory to this domain. Author: Drew Reisinger, Johns Hopkins University (reisinger@cogsci.jhu.edu).
} 
2.1. Doubly RELATIVE MODALITY. The standard doubly relative account of modality has its roots in Kratzer (1977), which introduces conversational backgrounds as a contextual parameter of modals, and is developed further in Kratzer (1981), which introduces ordering sources, but this paper will use the version found in Kratzer (1991). The denotations of possibility modals, here exemplified by can, and necessity modals, exemplified by should, are reproduced ${ }^{1}$ in (1).

$$
\begin{aligned}
& \text { a. } \llbracket \text { can } \rrbracket^{w, f, g}=\lambda p_{\langle s, t\rangle} \cdot \exists w^{\prime} \in \max _{\leq_{g(w}}[\cap f(w)]: p\left(w^{\prime}\right) \\
& \text { b. } \llbracket \text { should } \rrbracket^{w, f, g}=\lambda p_{\langle s, t\rangle} \cdot \forall w^{\prime} \in \max _{\leq_{g(w)}}[\cap f(w)]: p\left(w^{\prime}\right)
\end{aligned}
$$

The two denotations differ only in the quantifier over possible worlds. Both take a proposition $p$ as their argument, and both depend on two contextual parameters, $f$ and $g$, which are so-called conversational backgrounds, functions that map each possible world to a set of propositions. The conversational background $f$ is called the modal base, and intuitively, $f(w)$ is a set of propositions representing the content of some information state or body of evidence. For example, the propositions in $f(w)$ might be the beliefs of the speaker in $w$ or the mutual beliefs of the speaker and listener in $w$. The conversational background $g$ is called the ordering source. Intuitively, the propositions in $g(w)$ characterize some kind of ideal in $w$. For example, a deontic ordering source maps $w$ to a set of moral edicts or laws that hold in $w$, a teleological ordering source maps $w$ to a set of goals of some agent in $w$, and a stereotypical ordering source maps $w$ to a set of propositions characterizing what typically (but not always) happens in $w$ (see Kratzer 1981:45 for a more detailed discussion of different ordering source types). Thus, $\llbracket c a n \rrbracket^{w, f, g}(p)$ is true if and only if among the worlds consistent with some body of information $f(w)$ that best conform to the ideal defined by $g(w)$, there is at least one world in which $p$ is true. Similarly, 【should $\rrbracket^{w, f g}(p)$ is true if and only if $p$ is true in all of the $g(w)$-best worlds consistent with $f(w)$.

Formally, the notion of conforming to the ideal characterized by $g(w)$ is modeled by the preordering denoted $\leq_{g(w)}$ and defined as in (2).

$$
u \leq_{g(w)} v \text { iff }\{p \in g(w) \mid p(u)\} \subseteq\{p \in g(w) \mid p(v)\}
$$

That is, the world $v$ is at least as good as the world $u$ with respect to $g(w)$ if and only if every proposition in $g(w)$ satisfied by $u$ is also satisfied by $v$. The max operator in (1) picks out the maximal worlds in $\cap f(w)$ according to the ordering $\leq_{g(w)}$.

2.2. Combined ordering Sources And the Medicine Problem. Although the account in the previous section is standardly used in the modality literature, it has been challenged on several fronts. One such challenge comes from Goble's (1996) Medicine Problem, which was originally proposed as a problem for deontic logics but was adapted and simplified by Lassiter (2011) to argue against quantificational modal semantics.

The Medicine Problem scenario, in Lassiter's (2011) simplified form, is as follows. A patient is deathly ill, and a doctor must choose which of two medicines, A and B, to administer to the patient and cannot choose both. Medicine A is risky, as although it will completely cure the patient with low probability, it has a high probability of killing the patient. On the other hand, medicine B will always leave the patient alive and cure the disease, but its side effects will leave the patient somewhat debilitated. Given this scenario, many (including Goble 1996, Lassiter

\footnotetext{
${ }^{1}$ The denotations used here differ from Kratzer's (1991) in two respects. First, unlike Kratzer, I assume that there are maximal worlds in the ordering induced by $g(w)$ and hence use the max operator. Second, I use the notation $u \leq_{g(w)} v$ to mean that $v$ is at least as good a world as $u$ with respect to $g(w)$, whereas the notation is reversed in Kratzer (1991) and many subsequent papers.
} 
2011, and Katz et al. 2012) have the intuition that (3) is, or at least can be, true, as medicine B is less risky.

\section{(3) The doctor should administer medicine B.}

However, the standard account predicts unambiguously that the doctor should administer medicine A because the best worlds under an ordering source that values the eventual health of the patient are those improbable worlds in which the patient takes medicine A and survives, even though the worlds in which the patient takes medicine A and dies are worse than those in which medicine $\mathrm{B}$ is administered.

Katz et al. (2012), in agreement with Lassiter (2011), attributes the failure of the standard account in this case to its inability to model both the desirability and likelihood of outcomes with a single ordering source. However, contra Lassiter (2011), who argues for a probabilistic analysis of the modal operator, Katz et al. (2012) solve the Medicine Problem while preserving Kratzer's (1991) quantificational modal semantics by changing the ordering source in a systematic way. In particular, given an ordering source $g_{1}$ that orders worlds by their likelihood and an ordering source $g_{2}$ that orders them by their desirability, they construct a new ordering source $g_{1} * g_{2}$ using the operator defined in (4).

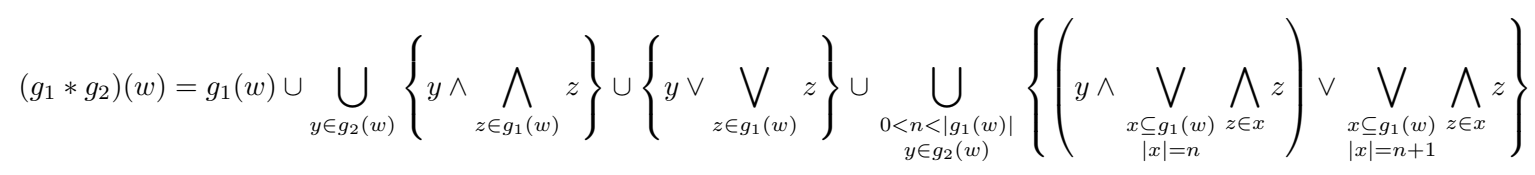

The * operator takes two ordering sources $g_{1}$ and $g_{2}$ as input and produces a new ordering source that, when combined with Kratzer's (1991) definition of $\leq_{g(w)}$, first orders worlds according to $g_{1}$ and then breaks any remaining ties according to $g_{2}$ (see Katz et al. 2012:497 for intuitions about why this definition works). In other words, $g_{1} * g_{2}$ is an ordering source that prioritizes $g_{1}$ over $g_{2}$. In the Medicine Problem scenario, if likelihood is prioritized over desirability, then the most desirable possible worlds after first restricting to the most likely worlds are indeed the worlds in which the patient takes medicine B. Even though the worlds in which they take A and survive are more desirable, they are not among the most likely worlds.

One advantage of this solution is that it accounts for the intuition that the truth value of (3) is subjective depending on the speaker's priorities. If the speaker indeed prioritizes likelihood over desirability, and hence uses the ordering source $g_{1} * g_{2}$, they judge (3) to be true, but if they prioritize desirability over likelihood, and hence use $g_{2} * g_{1}$, they judge it to be false. The formal machinery of Katz et al. (2012) indeed correctly predicts this dependence of the truth value on subjective priority. Nevertheless, it is precisely this dependence on subjective priority that motivates the puzzle I present in the next section.

3. The Embedded Medicine Problem. In this section, I propose the Embedded Medicine Problem (EMP), a slight extension of Lassiter's (2011) version of the original Medicine Problem that challenges the Katz et al. (2012) implementation of ordering source priority. As noted in the previous section, the truth value of (3), reproduced below as (5), depends on the speaker's subjective priority ranking between the likelihood and desirability ordering sources. Because of this subjectivity, sentence (6) can reasonably be uttered in a scenario in which the speaker and Kat disagree about how the two ordering sources should be ranked.

(5) The doctor should administer medicine B.

(6) The doctor should administer medicine B, but Kat thinks they should administer A. 
In fact, the disagreement in (6) can reasonably occur even if Kat and the speaker agree on both of the following pieces of contextual information: What the effects of the two different medicines could be and that the doctor cannot administer both medicines (the modal base), which outcomes are most desirable (the ordering source $g_{2}$ ), and how likely each outcome is given a choice of medicine (the ordering source $g_{1}$ ). That is, (6) is true in a scenario where the speaker's beliefs and Kat's beliefs differ minimally in the subjective degrees of priority assigned to the two relevant ordering sources.

Nevertheless, Katz et al.'s (2012) account predicts that (6) is false in the scenario described. In fact, if Kat and the speaker agree on the modal base and the ordering sources $g_{1}$ and $g_{2}$, it predicts that (6) is a contradiction. Formally, for Kat and the speaker to agree on the modal base means that in any world $w$ consistent with Kat's beliefs, $f(w)=f\left(w_{@}\right)$, where $w_{@}$ is the world of evaluation supplied by the speaker's context of utterance. If they agree on both of the ordering sources, then the same condition holds replacing $f$ with $g_{1}$ and $g_{2}$. Now if the ordering source used to interpret (6) is any function of $g_{1}$ and $g_{2}$, for example $g_{1} * g_{2}$ or $g_{2} * g_{1}$, then that ordering source will also be the same in $w @$ and in Kat's belief worlds. Thus, both occurrences of should in (6) quantify over the same set of possible worlds, $\max _{\leq_{g\left(w_{@}\right)}}\left[\cap f\left(w_{@}\right)\right]$. But then the first conjunct of (6) implies that every world in this set is one in which the doctor administers medicine $\mathrm{B}$, and the second conjunct implies that in every world in this set, the doctor administers A. Since the modal base eliminates worlds in which the doctor administers both medicines, the two conjuncts of (6) are predicted to be inconsistent, contrary to the intuitions in the previous paragraph.

Intuitively, Katz et al.'s (2012) account could accommodate the EMP if the modal in the first conjunct could be interpreted with respect to $g_{1} * g_{2}$, and the second modal could be interpreted with respect to $g_{2} * g_{1}$. However, the machinery of this account provides no way to shift the ordering source in the embedded context. Rather, Katz et al. (2012) envisions the * operator as a way of deriving the ordering source required to interpret an utterance from more intuitive ordering sources, such as simple likelihood and desirability ordering sources, that are accessible in context. In the next section, I rectify this limitation of this formalism by moving ordering source priority into the core semantics of modality.

4. Ranked ordering sources. In this section, I first describe the primary formal contribution of this paper, ranked ordering sources. I then use this formalism to address the difficulties raised by the EMP. Afterward, I introduce notation based on Optimality Theory to facilitate manual computation with ranked ordering sources.

4.1. INTRODUCING PARTIAL ORDERS. As described previously, ordering sources as proposed by Kratzer (1981) are functions from possible worlds to sets of propositions, and these propositions determine an ordering on worlds. In particular, all of the propositions in the ordering source are weighted equally in constructing the order on worlds.

The following slightly modified example from Katz et al. (2012) illustrates this property and shows why it may be undesirable. If $g$ is an ordering source representing the laws of some jurisdiction in a given possible world, then in the actual world, $g\left(w_{@}\right)$ would probably include $\{\lambda w$. There is no murder in $w, \lambda w$. There is no jaywalking in $w\}$. The ordering on possible worlds induced by this ordering source is represented in the Hasse diagram in Figure 1, in which worlds toward the top of the diagram are ordered above those toward the bottom of the diagram. 


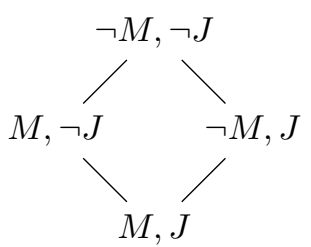

Figure 1. The ordering on worlds induced by the flat ordering source $g\left(w_{@}\right)=\{\neg M, \neg J\}$, where $M$ and $J$ denote the propositions that there is murder and jaywalking, respectively, in $w$.

According to this order, the best worlds are those in which neither of the crimes occur, and the worst are those in which both crimes occur. However, the worlds in which only one of the crimes occurs are no better or worse than the other. This ordering source disprefers murder just as much as it does jaywalking, contrary to intuitions. Ideally, the ordering source should be able to represent both that murder and jaywalking violate the law and that murder is a worse violation than jaywalking. Like Katz et al. (2012), I argue that examples like this and the Medicine Problem motivate formal models of ordering source priorities, but rather than implementing such priorities with a contextual operator on ordering sources, I propose to encode priorities explicitly in the ordering sources themselves.

Formally, a ranked ordering source is a function from possible worlds to partially ordered sets of propositions. That is, a ranked ordering source not only contains propositions that define some ideal but also an irreflexive and transitive relation $\prec$ on those propositions that ranks them by their weight in contributing to the ideal. ${ }^{2}$ I will use the notation $g, \prec$ to denote a ranked ordering source and $g(w), \prec_{w}$ to denote a ranked ordering source evaluated at a particular world. In the crime example above, an intuitive choice of ranked ordering source would be $g(w)=\{\neg M, \neg J\}$ such that $\neg J \prec_{w} \neg M$. That is, not murdering is more important to the ideal than not jaywalking.

Although the addition of partial order structure to ordering sources does intuitively capture how different propositions in the ordering source can have different weights, I have not yet specified how a partially ordered set, or poset, of propositions induces an ordering on possible worlds. First, I will develop the intuition for my definition. According to Kratzer's (1991) ordering, $v$ is at least as good as $u$ with respect to an unranked ordering source $g(w)$ if every proposition in $g(w)$ satisfied at $u$ is also satisfied at $v$. Equivalently, $v$ is at least as good as $u$ if every proposition in $g(w)$ that is not satisfied at $v$ is also not satisfied at $u$. Thus, testing whether $v$ is at least as good as $u$ can be thought of in terms of the violations by $v$ of the ideal: If $v$ has any violations that $u$ does not, then $v$ is not at least as good as $u$. In the crime example, if $v$ is a world with jaywalking and no murder, and $u$ is a world with murder and no jaywalking, then $v$ is not better than $u$ because it has a violation, namely jaywalking, that $u$ does not.

However, intuition suggests that $v$ 's violation of the ideal is forgivable relative to $u$ because $u$ has an even worse violation. This motivates a new test for whether $v$ is better than $u$ : If $v$ has any violations that $u$ does not, and those violations are not overshadowed by an even worse violation of $u$ 's, then $v$ is not at least as good as $u$. This test is formalized in (7), where $V_{g(w)}(u)(\mathrm{read}$ " $u$ 's violations in $g(w)$ ") denotes the subset $\{p \in g(w) \mid \neg p(u)\}$ of propositions in $g(w)$ that are false at the possible world $u$.

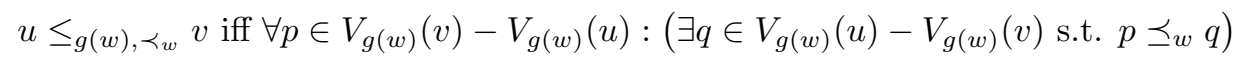

\footnotetext{
2 Throughout the paper, I will use the straight ordering symbol $<$ to denote orderings on possible worlds and the curved ordering symbol $\prec$ to denote orderings on ordering source propositions.
} 
That is, $v$ is at least as good as $u$ with respect to a poset of propositions $g(w), \prec_{w}$ if and only if for every one of $v$ 's violations that $u$ doesn't have, $u$ has a higher-ranked violation that $v$ doesn't have. Applying the order in (7) to the ranked ordering source defined above, in which no murder is ranked above no jaywalking, yields the ordering on worlds in Figure 2.

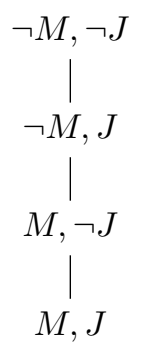

Figure 2. The ordering on worlds induced by the ranked ordering source $g(w)=\{\neg M, \neg J$ such

$$
\text { that } \neg J \prec_{w} \neg M \text {. }
$$

According to this ordering, the best worlds are those with neither crime, followed by the jaywalking worlds, then the murder worlds, and finally the worlds with both crimes.

4.2. Solving the Embedded Medicine Problem. I now show how the ranked ordering sources developed in the previous section can be used to account for the logical consistency of (6), reproduced below as (8), even when the speaker and Kat agree on the modal base and ordering source propositions.

(8) The doctor should administer medicine B, but Kat thinks they should administer A.

In order to be more precise about the contents of the modal base and ordering source, let $A$ and $B$ denote the propositions that the doctor administers medicine A or B, respectively, let alive denote the proposition that the patient survives their treatment, let healthy denote the proposition that the patient suffers no side effects as a result of the treatment, and let responsive denote the proposition that the patient would respond positively to medicine A. The modal base should capture facts such as The doctor cannot administer both drugs, Medicine B will cause the patient to be alive but not healthy, and If the patient is not responsive to medicine A, then administering it will cause the patient to die. An appropriate modal base is shown in (9), and since Kat and the speaker of (8) agree on these facts, the modal base has this value both in the world of evaluation $w_{@}$ and Kat's belief worlds.

$$
\begin{aligned}
f(w)=\{ & \neg(A \wedge B), A \vee B, B \rightarrow(\text { alive } \wedge \neg \text { healthy }),(A \wedge \text { responsive }) \rightarrow(\text { alive } \wedge \text { healthy }), \\
& (A \wedge \neg \text { responsive }) \rightarrow \neg \text { alive }\}
\end{aligned}
$$

Just as in Katz et al.'s (2012) solution to the Medicine Problem, the ordering source should contain information about what is likely. Since it is more likely than not that the patient will not respond well to medicine $A, g(w)$ should contain $\neg$ responsive. Furthermore, it should contain information about what is desirable, so $g(w)$ should contain alive and healthy. Since Kat and the speaker agree about what is likely and what is desirable, $g(w)$ should contain the same propositions in $w_{@}$ and in Kat's belief worlds.

Finally, the ordering source also has a priority order $\prec_{w}$ on the propositions. Since the speaker thinks the doctor should administer B, the likelihood proposition $\neg$ responsive should be prioritized over the desirability propositions alive and healthy in $w_{@}$. Since Kat prioritizes desirability over likelihood, the order is inverted in her belief worlds. The ranked ordering source evaluated at $w_{@}$ and at any of Kat's belief worlds is presented in Hasse diagram form in Figure 3. 

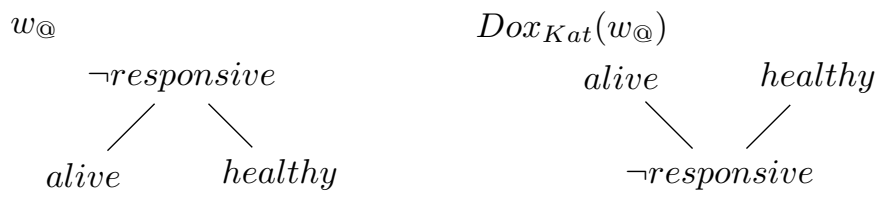

Figure 3. The ranked ordering source $g(w), \prec_{w}$ for the EMP evaluated at the world of evaluation supplied by the utterance context (left) and at any of Kat's doxastically accessible worlds (right).

Now that the contextual parameters are fixed, the truth conditions of (8) can be computed. The first conjunct is true if and only if the doctor administers medicine B in all of the highest ranked worlds, according to the speaker, that are consistent with the modal base. Similarly, the second conjunct is true if and only if the doctor administers medicine $\mathrm{A}$ in all of the highest ranked worlds, according to Kat, that are consistent with the modal base. Applying the definition of $\leq$ in (7) to the ranked ordering sources in Figure 3 yields the orderings over possible worlds in Figure 4 .

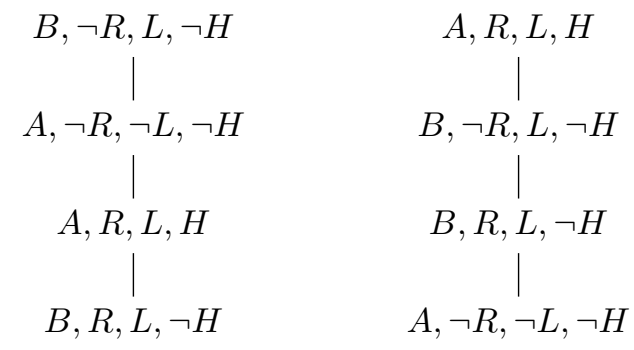

Figure 4. The orderings over worlds induced by the ranked ordering sources in Figure 3 . The ordering on the left is induced by the ranked ordering source evaluated at $w_{@}$, and the one on the right is induced by the ranked ordering source in Kat's belief worlds. These diagrams use the following abbreviations: $R=$ responsive, $L=$ alive, $H=$ healthy.

Because the speaker prioritizes likelihood over desirability, the worlds in which the patient responds poorly to medicine A are ranked above those in which they respond well to it. Among the not-responsive worlds, the worlds in which the patient takes medicine B (and survives with side effects) are ranked above those in which the patient takes A (and dies) according to desirability. Among the less likely responsive worlds, the $A$ worlds (in which the patient recovers fully) are ranked above the $B$ worlds. Most importantly, the maximal worlds are all $B$ worlds, so the first conjunct of (8) is true.

In contrast, since Kat prioritizes desirability over likelihood, the responsive $A$ worlds are ranked above all of the $B$ worlds, which are in turn ranked above the not-responsive $A$ worlds. Among the $\mathrm{B}$ worlds, the not-responsive worlds are ranked above the responsive worlds by likelihood. Since the highest ranked worlds (in all of Kat's doxastically accessible worlds) are all $A$ worlds, the second conjunct of (8) is also true, and hence the entire sentence is predicted to be true in accordance with intuition. In particular, because the ranked ordering source formalism allows the relative priority of the likelihood and desirability ordering sources to vary with the world of evaluation, this account does not predict that (8) is a contradiction.

4.3. EASier ORDERING With Optimality TheORY tableauX. Although the definition in (7) correctly generalizes Kratzer's (1991) ordering semantics to partially ordered ordering sources, it is somewhat cumbersome to work with by hand, limiting its usefulness to practicing semanticists who need to predict the truth values of modal sentences. Fortunately, given a mild technical as- 
sumption, ${ }^{3}$ computing the maximal worlds under this ordering turns out to be equivalent to optimizing a certain Optimality Theory tableau that is significantly easier to manipulate for those familiar with the formalism.

The machinery of Optimality Theory (Prince \& Smolensky 2008) is primarily used in phonology and syntax and provides a framework for optimizing linguistic structures with respect to a set of ranked, violable grammatical constraints. My use of this formalism does not carry most of its theoretical commitments (e.g., the universality of the constraint set), but it exploits the fact that optimizing a tableau and computing the maximal worlds under the ordering in (7) are algorithmically similar. Given a ranked ordering source $g(w), \prec_{w}$ and modal base $f(w)$, the maximal worlds in the modal base can be computed by optimizing a tableau with the possible worlds in $\cap f(w)$ as its candidate set and the propositions in $g(w)$ as its constraints, ranked by $\prec_{w}$. A world $v$ in $\cap f(w)$ is said to violate a constraint $p$ in $g(w)$ if $p$ is false at $v$. For example, the tableau used to derive the speaker's ordering on worlds in the EMP is shown in Figure 5.

\begin{tabular}{|r||c|c:c|}
\hline World & $\neg$ responsive & alive & healthy \\
\hline \hline$B, \neg R, L, \neg H$ & & $*$ & $*$ \\
$A, \neg R, \neg L, \neg H$ & & $*$ & $*$ \\
$A, R, L, H$ & $*$ & & $*$ \\
$B, R, L, \neg H$ & $*$ & & $*$ \\
\hline
\end{tabular}

Figure 5. The Optimality Theory tableau used to derive the speaker's ordering on worlds in the EMP. Asterisks denote constraint violations, and the pointing hand indicates which candidate (possible world) is optimal with respect to the constraints (ordering source). The dashed column separator indicates that the alive and healthy constraints are ranked at the same level.

Optimization in a tableau proceeds by treating each constraint in order based on its ranking. If some candidates violate a constraint and others do not, then those that violate that constraint are eliminated. This continues either until a single candidate remains or until all of the constraints are considered, in which case all of the remaining candidates are maximal. This optimization differs from standard Optimality Theory in that blocks of equally ranked constraints can occur, such as alive and healthy in Figure 5. These blocks are treated as a single constraint, and candidates are compared on this constraint using the subset relation between their violation sets. For example, the first row of Figure 5 is better than the second with respect to the alive and healthy constraint block because the violations of the first row are a proper subset of the violations of the second row.

At this point, I do not wish to imply any deeper connection between the theory of grammar that usually accompanies Optimality Theory and ranked ordering source semantics. Rather, Optimality Theory tableaux are simply a convenient notation for communicating ranked ordering sources and the orderings on worlds that they induce.

5. The priority join and the structure of modality. Although the ranked ordering source solution in the previous section avoids some of the problematic predictions of Katz et al.'s (2012) account for the EMP, it also gives up a desirable theoretical and methodological property. Namely, whereas the complex ordering source $g_{1} * g_{2}$ is constructed from simpler ordering sources that are intuitively accessible from the context, the ranked ordering source that I proposed is some-

\footnotetext{
${ }^{3}$ Namely, this is only possible if the ranked ordering source is a graded poset. Roughly, a poset is graded if its elements can be assigned to discrete "levels" or "tiers" that are consistent with the ordering. I do not yet know of any examples of modals that require a non-graded ranked partial order.
} 
what ad hoc. It cannot be described as just a likelihood or desirability ordering source, as it contains propositions from both ideals. Furthermore, although the particular partial order $\prec_{w} \mathrm{I}$ proposed is reasonable, many other less intuitive partial orders are possible, such as healthy $\prec_{w}$ $\neg$ responsive $\prec_{w}$ alive. This is theoretically undesirable because the ranked ordering source formalism seems to overgenerate possible truth conditions for sentences containing modals, as each unranked ordering source admits many possible partial orders. ${ }^{4}$ Additionally, the lack of constraints on ranked ordering sources creates a difficult problem for listeners: determining which of the many possible ranked ordering sources was intended by the speaker of a modal statement.

In contrast, Katz et al. (2012) mitigates these problems by imposing algebraic structure on the space of ordering sources. In fact, one of the primary motivations for their approach is the observation that "there is not always a clear, direct relationship between the propositions that we are using to order worlds and those that we refer to when we describe the context for a modal utterance" (Katz et al. 2012:495). Thus, operators like * explain the relationship between intuitively accessible descriptions of contexts, such as those that can be paraphrased with "in view of" statements (e.g. "in view of what is likely", "in view of what the law allows"), and the often more complex ordering sources that are actually required to capture observed linguistic data as in the case of the Medicine Problem. Such operators also mitigate the overgeneration problem by restricting attention to the subset of possible ordering sources that are derivable from a collection of intuitively accessible primitives, such as deontic or teleological ordering sources, and a small set of combinatorial operators on those primitives, such as the * operator. Finally, if listeners also only consider such a structured subspace of ordering sources, then this algebraic structure constrains theories of how listeners resolve the vagueness of modal statements.

In order to retain these desirable properties of Katz et al.'s (2012) account, I propose a generalization of the * operator, called the priority join and denoted $\sqcup$, that takes two ranked ordering sources as input and prioritizes one over the other in a world-dependent way. It also takes an additional parameter PRI, which is a function that takes a possible world $w$ as input and returns the index ( 1 or 2 ) of the input ordering source with the higher priority in $w$. The precise definition is given in (10).

$$
\begin{aligned}
& \left(\left(g_{1}, \prec_{1}\right) \sqcup_{\mathrm{PRI}}\left(g_{2}, \prec_{2}\right)\right)(w)=\left(g_{1}(w) \cup g_{2}(w), \prec_{w}^{\prime}\right) \\
& \text { where } p \prec_{w}^{\prime} q \text { iff } p \prec_{1, w} q \vee p \prec_{2, w} q \vee\left(p \notin g_{\mathrm{PRI}(w)}(w) \wedge q \in g_{\mathrm{PRI}(w)}(w)\right)
\end{aligned}
$$

That is, given two ranked ordering sources $g_{1}, \prec_{1}$ and $g_{2}, \prec_{2}$ and function PRI, their priority join is a new ranked ordering source that contains all of the propositions in $g_{1}$ and $g_{2}$, preserves the partial orders $\prec_{1}$ and $\prec_{2}$ within $g_{1}$ and $g_{2}$, and ranks all of the propositions in one of the input ordering sources over those in the other depending on the value of PRI. For example, if PRI $(w)=$ 1 , then all of the propositions in $g_{1}(w)$ are ranked above those in $g_{2}(w)$ in the priority join. The * operator of Katz et al. (2012) is equivalent to a special case of the priority join in which $g_{1}$ and $g_{2}$ are unranked and $\operatorname{PRI}(w)=1$ in every world $w$.

Using this operator, the ranked ordering source I used to account for the EMP in the previous section can be derived in a principled way. Let $g_{1}(w)=\{\neg$ responsive $\}$ and $g_{2}(w)=\{$ healthy,

\footnotetext{
${ }^{4}$ In fact, for any ranked ordering source, there is an equivalent unranked ordering source that induces the same ordering on possible worlds, so strictly speaking, the ranked ordering source account does not overgenerate relative to the standard account. Nevertheless, if the unranked ordering sources are restricted to an intuitively accessible subset, such as those expressible using "in view of" phrases, the ranked ordering source account does overgenerate with respect to that restricted subset.
} 
alive be the likelihood and desirability ordering sources, respectively, where $w$ is the utterance evaluation world or any of Kat's belief worlds. In addition, let PRI( $\left.w_{@}\right)=1$ in the evaluation world, since the speaker prioritizes likelihood, and let PRI $(w)=2$ in Kat's belief worlds, since she prioritizes desirability. Then the EMP ranked ordering source is $g_{1} \sqcup_{\mathrm{PRI}} g_{2}$. Thus, the priority join solves the problems raised by the EMP while retaining virtues of Katz et al.'s (2012) account by deriving the more complex ordering source required to capture judgments from simpler, intuitively accessible ordering sources.

6. Conclusion and future work. In this paper, I have presented the Embedded Medicine Problem, which suggests that ordering source priorities are not just fixed at the level of the utterance and can be manipulated by intensional operators such as attitude verbs. I have introduced ranked ordering sources, which allow ordering source propositions to have varying degrees of importance in determining an ordering on possible worlds, and I have shown how the worlddependent priority afforded by the ranked ordering source account avoids the problems that the Embedded Medicine Problem raises for Katz et al.'s (2012) account of ordering source priority. Finally, in the spirit of the proposal by Katz et al. (2012) that complex ordering sources required to account for data should be explicitly related to the simpler and intuitively accessible ordering sources that semanticists use to characterize discourse context, I have proposed the priority join operator, which combines two ranked ordering sources using a world-dependent priority relation.

If ranked ordering sources correctly model how contextually supplied priorities affect modal interpretation, then one would expect evidence of this additional structure in different domains of modality. I will speculate on a few such domains here, though the details remain to be developed. Firstly, one of the chief motivations for Katz et al.'s (2012) project of developing ordering source operators was to understand the semantics of gradable modal expressions (GMEs), or modal expressions that can occur in comparative constructions and with degree modification. In some cases, I suspect, the degree scale manipulated by these constructions may be derived in part from the partial order on the ranked ordering source. For example, the truth of the comparative statement in (11) is arguably related to the relative priority of the propositions $\neg$ murder and jjaywalk in the ordering source used to interpret the modal adjective worse.

(11) It is worse to commit murder than to jaywalk.

Another potential application of ranked ordering sources comes from Rubinstein's (2012) account of weak necessity modals. Rubinstein (2012) makes a distinction between two different kinds of ordering source: primary ordering sources, whose propositions are collectively committed to as desirable by all conversational participants, and secondary ordering sources, which are not committed to as desirable by at least one participant. The difference between strong necessity modals like have to and weak necessity modals like ought to is that strong necessity modals are interpreted only with respect to the primary ordering sources, and weak necessity modals are also interpreted with respect to some secondary ordering source. Furthermore, when interpreting weak necessity modals, the domain of possible worlds is first restricted by the primary ordering sources before optimizing with respect to the secondary ordering source. This is functionally equivalent to assigning the secondary ordering source a lower priority, in the sense of ranked ordering source priority, than the primary ordering sources. If the distinction between primary and secondary ordering sources is best modeled using ranked ordering sources, then one should expect that an ordering source's primary or secondary status may be shifted by intentional operators. Thus, examples of weak necessity models that are (or aren't) contextually licensed in a 
matrix clause but aren't (or are) licensed in the scope of some intentional operator would be additional converging evidence in favor of ranked ordering sources.

Yet another semantic formalism that may bear on ranked ordering sources comes from Villalta's (2008) account of predicates that license the subjunctive mood in Spanish. According to Villalta (2008), the semantic commonality between subjunctive-licensing predicates is that they involve comparing the proposition denoted by their complement clause to a set of alternative propositions along some scale, such as desirability or likelihood. In particular, the ordering on the set of alternative propositions is derived from a primitive ordering on worlds. If $<_{x, w}$ is the desirability ordering on worlds for an entity $x$ in world $w$ - that is, $u<_{x, w} v$ if and only if $v$ is a more desirable world to $x$ in $w$ than $u$-then an ordering $<_{\mathrm{DES} x, w}$ on propositions is defined as in (12).

(12) $p \leq_{\mathrm{DES} x, w} q$ iff for all $u$ such that $p(u)$, there exists $v$ such that $q(v)$ and $u<_{x, w} v$

Interestingly, the relationship between $<_{\mathrm{DES} x, w}$ and $<_{x, w}$ is very similar to that between the partial order $\prec_{w}$ on a ranked ordering source and the ordering on possible worlds in induces. In fact, if an ordering on propositions $\prec$ induces an ordering $<$ on possible worlds as a ranked ordering source, and $\prec_{\mathrm{DES}}$ is an ordering on propositions derived from $<$ using (12), then $\prec=\prec_{\mathrm{DES}}$. Thus, Villalta's (2008) construction is a left inverse of the function that maps ranked ordering sources to induced orderings on possible worlds. It is an open question whether the partial orders of primitive ranked ordering sources, such as likelihood and desirability, can all be so derived from the world orderings implicated in the semantics of subjunctive-licensing predicates. In addition, Villalta (2008) proposes a degree semantics for subjunctive predicates that derives from the ordering $<_{\mathrm{DES} x, w}$ on alternative propositions. It remains to be seen whether the same degree scale constructed from ranked ordering sources can give a satisfactory account of GME's such as the one in (11).

\section{References}

Goble, Lou. 1996. Utilitarian deontic logic. Philosophical Studies 82(3). 317-357. http://dx.doi.org/10.1007/BF00355312.

Katz, Graham, Paul Portner \& Aynat Rubinstein. 2012. Ordering combination for modal comparison. Semantics and Linguistic Theory (SALT) 22. 488-507. http://dx.doi.org/10.3765/salt.v22i0.2647.

Kratzer, Angelika. 1977. What 'must' and 'can' must and can mean. Linguistics and Philosophy 1(3). 337-355. http://dx.doi.org/10.1007/BF00353453.

Kratzer, Angelika. 1981. The notional category of modality. In Hans Jürgen Eikmeyer \& Hannes Rieser (eds.), Words, Worlds, and Contexts (Research in Text Theory 6). 38-74. Berlin, Boston: De Gruyter.

Kratzer, Angelika. 1991. Modality. In Arnim von Stechow \& Dieter Wunderlich (eds.), Semantics: An international handbook of contemporary research (Handbooks of Linguistics and Communication Science 6). 639-650. Berlin, Boston: De Gruyter Mouton.

Lassiter, Daniel. 2011. Measurement and modality: The scalar basis of modal semantics. New York: New York University dissertation.

Prince, Alan \& Paul Smolensky. 2008. Optimality Theory: Constraint interaction in generative grammar. Hoboken, NJ: John Wiley \& Sons.

Rubinstein, Aynat. 2012. Roots of modality. Amherst, MA: University of Massachusetts Amherst dissertation. 
Villalta, Elisabeth. 2008. Mood and gradability: An investigation of the subjunctive mood in Spanish. Linguistics and Philosophy 31(4). 467-522.

http://dx.doi.org/10.1007/s 10988-008-9046-x. 